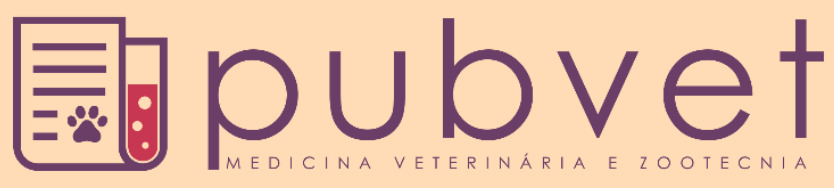

https://doi.org/10.31533/pubvet.v15n03a761.1-9

\title{
Adenocarcinoma pulmonar com metástase hepática em gato: Relato clínico, radiológico, anatomopatológico e citopatológico
}

\author{
João Felipe Rito Cardoso ${ }^{*} \bullet$, Roberta Salim Menezes $^{2} \bullet$, Mariana Moura ${ }^{3} \bullet$, Elan Cardozo \\ Paes de Almeida ${ }^{4} \bullet$ \\ ${ }^{I}$ Médico veterinário Professor da Escola de Medicina Souza Marques (FTESM), Departamento de Morfologia Funcional, Rio de Janeiro -RJ, Brasil. \\ ${ }^{2}$ Médica Veterinária autônoma patologista, Rio de Janeiro - RJ, Brasil. \\ ${ }^{3}$ Médica Veterinária autônoma VetCat, Rio de Janeiro - RJ, Brasil. \\ ${ }^{4}$ Médica Veterinária Professora do Instituto de Saúde de Nova Friburgo. Universidade Federal Fluminense, Nova Friburgo - RJ, Brasil \\ *Autor de Correspondência: E-mail: ifrcardoso@gmail.com
}

Resumo. A incidência de neoplasias pulmonares primárias em gatos vem aumentando devido a muitos fatores, entre eles o maior contato com poluentes atmosféricos. Entretanto, o diagnóstico se faz tardiamente na maioria dos casos, impossibilitando a adoção de terapias eficientes. Nesse sentido, o presente relato apresenta a comparação clínica, radiológica, anatomopatológica e citopatológica de um adenocarcinoma pulmonar com metástase hepática, visando fornecer informações úteis ao clínico para a triagem e detecção precoce desta condição utilizando ferramentas de baixo custo, fácil execução, pouco invasivas e confiáveis. O presente relato mostra sintomas de perda ponderal progressiva, anorexia, letargia e dispneia leve, associados a presença de massa circunscrita radiopaca em lobo pulmonar caudal direito e opacificação pulmonar intersticial difusa, ao raio X. Na necropsia, presença de efusões em cavidades torácicas e abdominais e massas tumorais em pulmão e fígado. O exame citopatológico sugeriu diagnóstico de carcinoma, sendo corroborado pelo diagnóstico anatomopatológico de adenocarcinoma pulmonar misto grau 2, com metástase hepática. As comparações realizadas neste estudo sugerem que o exame radiológico é essencial para o diagnóstico e estadiamento clínico de neoplasias pulmonares, ao passo que a histopatologia é o critério ouro para o diagnóstico definitivo e classificação tumoral. Contudo, a citopatologia é uma ferramenta diagnostica rápida, pouco invasiva e segura para o diagnóstico preliminar e durante atos cirúrgicos e necroscópicos quando não se tem acessível histopatologia por congelação ou laboratórios de patologia.

Palavras-Chave: Citopatologia, felino, neoplasia, pulmão

\section{Pulmonary adenocarcinoma with liver metastasis in a cat: Clinical, radiological, anatomopathological and cytopathological report}

Abstract. The incidence of primary lung cancer in cats has been increasing due to many
factors, including greater contact with air pollutants. However, the diagnosis is made late
in most cases, making it impossible to adopt efficient therapies. In this sense, the present
report presents the clinical, radiological, anatomopathological and cytopathological
comparison of a pulmonary adenocarcinoma with liver metastasis, aiming to provide useful
information to the clinician and early screening detection, as well as using low cost, easy-
to-perform, low-invasive and reliable tools. The present report shows symptoms of
progressive weight loss, anorexia, lethargy and mild dyspnea, associated with the presence
of a circumscribed radiopaque mass in the right caudal pulmonary lobe and diffuse
interstitial pulmonary opacification at X-ray. At necropsy, presence of effusions in the
thoracic and abdominal cavities and tumor masses in lung and liver. The cytopathological 
examination suggested a diagnosis of carcinoma, being corroborated by the anatomopathological diagnosis of grade 2 mixed pulmonary adenocarcinomas, with liver metastasis. The comparisons made in this study suggest that radiological examination is essential for the diagnosis and clinical staging of lung neoplasms, while histopathology is the gold criterion for definitive diagnosis and tumor classification. However, cytopathology is a quick, low-invasive and safe diagnostic tool for preliminary diagnosis and during surgical and necroscopic procedures when freezing histopathology or pathology laboratories are not accessible.

Keywords: Cat, citopatology, lung, neoplasia

\title{
Adenocarcinoma pulmonar con metástasis hepática en gato: Informe clínico, radiológico, anatomopatológico y citopatológico
}

\begin{abstract}
Resumen. La incidencia de cáncer de pulmón primario en gatos ha ido en aumento debido a muchos factores, incluido un mayor contacto con los contaminantes del aire. Sin embargo, el diagnóstico se realiza tarde en la mayoría de los casos, lo que imposibilita la adopción de terapias eficaces. En este sentido, el presente informe presenta la comparación clínica, radiológica, anatomopatológica y citopatológica de un adenocarcinoma de pulmón con metástasis hepática, con el objetivo de brindar información útil al clínico para el cribado y detección precoz de esta patología mediante métodos de bajo costo, fácil realización, baja invasión y de confianza. El presente reporte muestra síntomas de pérdida progresiva de peso, anorexia, letargo y disnea leve, asociados a la presencia de una masa radiopaca circunscrita en el lóbulo pulmonar caudal derecho y opacificación pulmonar intersticial difusa, en la radiografía. En la necropsia, presencia de efusiones en las cavidades torácica y abdominal y masas tumorales en pulmón e hígado. El examen citopatológico sugirió el diagnóstico de carcinoma, siendo corroborado por el diagnóstico anatomopatológico de adenocarcinoma pulmonar mixto grado 2, con metástasis hepática. Las comparaciones realizadas en este estudio sugieren que el examen radiológico es fundamental para el diagnóstico y la estadificación clínica de las neoplasias pulmonares, mientras que la histopatología es el criterio de oro para el diagnóstico definitivo y la clasificación tumoral. Sin embargo, la citopatología es una herramienta de diagnóstico rápida, poco invasiva y segura para el diagnóstico preliminar y durante los procedimientos quirúrgicos y necroscópicos cuando no es accesibles la histopatología por congelación o laboratorios de patología.
\end{abstract}

Palabras clave: Citopatología, felino, neoplasia, pulmón

\section{Introdução}

A incidência de neoplasias pulmonares primárias em gatos vem aumentando, em decorrência do aumento da expectativa de vida, da melhora nas técnicas de diagnóstico e pelo maior contato com poluentes atmosféricos (Corgozinho et al., 2015; Travis et al., 2013). Apesar das causas indeterminadas, teorias apontam para mutações causadas por agentes virais ou produtos químicos, como fumaça de cigarro (Wilson, 2016).

As neoplasias primárias podem surgir do epitélio de condução ou do parênquima alveolar, predominando os tumores epiteliais de origem hilar ou bronco-alveolar. Entretanto, tumores mesenquimais primários também podem ser encontrados (Dungworth, 1999; Van der Linde-Sipman \& van den Ingh, 2000; Lopez, 2013). Os carcinomas pulmonares acometem gatos adultos (Lopez, 2013) com sinais e sintomas inespecíficos, como perda de peso, letargia, vômitos, além de manifestações respiratórias, como dispneia, taquipneia e tosse, relacionadas a presença de efusões pleurais, da própria massa tumoral ou invasão do tumor, que impedem a expansão pulmonar (Magalhães et al., 2001; Nunley et al., 2015; Lopez, 2013). Adenocarcinomas pulmonares são geralmente agressivos com predileção para metástases intrapulmonares, porém, diversos órgãos podem ser afetados, como órgãos abdominais (Caswell \& Williams, 2016; Corgozinho et al., 2015). 
O presente artigo tem como objetivo apresentar um relato de caso de adenocarcinoma pulmonar em uma gata com metástase hepática apresentando uma comparação dos achados clínicos, radiológicos, anatomopatológicos e citopatológicos.

\section{Relato de caso}

Felino, fêmea, 16 anos, pelo curto brasileiro, apresentando perda ponderal progressiva, anorexia, letargia e dispneia leve.

Realizada radiografia torácica, evidenciando a presença de massa circunscrita radiopaca com limites e contornos definidos, medindo 4,0 x 3,0 cm de diâmetro em lobo pulmonar caudal direito; opacificação pulmonar intersticial difusa, predominante em lobos caudais. Os achados radiográficos são sugestivos de neoplasia pulmonar e broncopatia moderada, como apresentado na Figura 1.

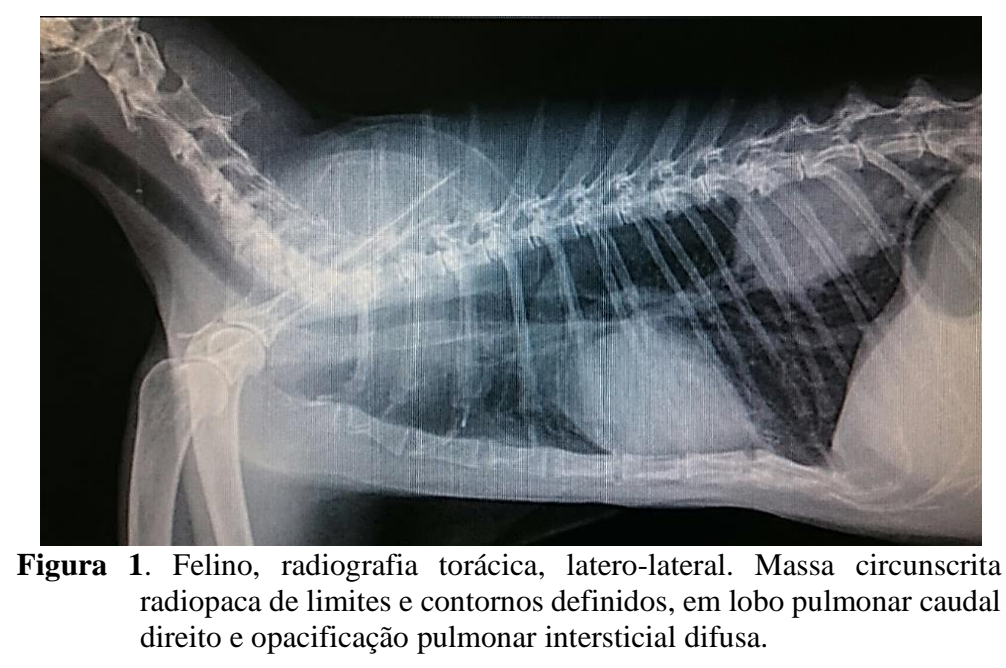

Após tratamento paliativo sem melhora do quadro clínico foi submetida à eutanásia, por determinação da proprietária e de acordo com normatização do CFMV 2013 (Aragão, 2012) e posterior exame anatomopatológico.

Durante a necropsia, ambas as cavidades torácica e abdominal apresentavam efusão sanguinolenta (Tabela 1), contendo aproximadamente $24 \mathrm{~mL}$ e $20 \mathrm{~mL}$, respectivamente.

Tabela 1. Comparação dos achados pulmonares

\begin{tabular}{|c|c|c|c|}
\hline Radiológicos & Macroscópicos & Histopatológicos & Cit \\
\hline $\begin{array}{l}\text { Opacificação pulmonar } \\
\text { intersticial difusa }\end{array}$ & Efusão torácica sanguinolenta & Não avaliado & aliado \\
\hline $\begin{array}{l}\text { inscrita radiopa } \\
\text { adal direito }\end{array}$ & $\begin{array}{l}\text { Nódulos multifocais a } \\
\text { coalescentes com superfície } \\
\text { multilobada por vezes cística } \\
\text { contendo muco acometendo } \\
\text { 2/3 do lobo caudal direito } \\
\end{array}$ & $\begin{array}{l}\text { Células epiteliais cúbicas com } \\
\text { moderada anisocariose em arranjos } \\
\text { trabeculares ou densos agregados } \\
\text { celulares, com fundo contendo } \\
\text { material sugestivo de muco. }\end{array}$ & $\begin{array}{l}\text { Células epiteliais com } \\
\text { moderado polimorfismo } \\
\text { em arranjos sólidos, } \\
\text { trabeculares, tubulares ou } \\
\text { acinares } \\
\end{array}$ \\
\hline
\end{tabular}

Os pulmões apresentavam nódulos multifocais a coalescentes, brancacentos acometendo aproximadamente $2 / 3$ do lobo pulmonar caudal direito, aos cortes superfície multilobada, firme-elástica, brancacenta, por vezes com cavidade cística contendo muco, como observado na Figura 2.

Fígado com aderência ao diafragma, com nódulos e massas multifocais à coalescente, firmeelásticos, multilobados, brancacentos, por vezes, císticos; acometendo diferentes lobos hepáticos, como observado na Figura 3. Os demais órgãos não apresentavam alterações dignas de nota.

Amostras citológicas do pulmão e fígado foram coletadas por punção aspirativa por agulha fina (PAAF) durante o procedimento, secas em ar ambiente, fixadas com metanol e coradas com solução rápida tipo Romanovsky. 
Amostras de todos os órgãos foram coletadas para exame histopatológico, sendo mantidas em solução fixadora de formalina neutra a $10 \%$, e posteriormente submetidas a processamento histológico de rotina e coloração com Hematoxilina e Eosina.

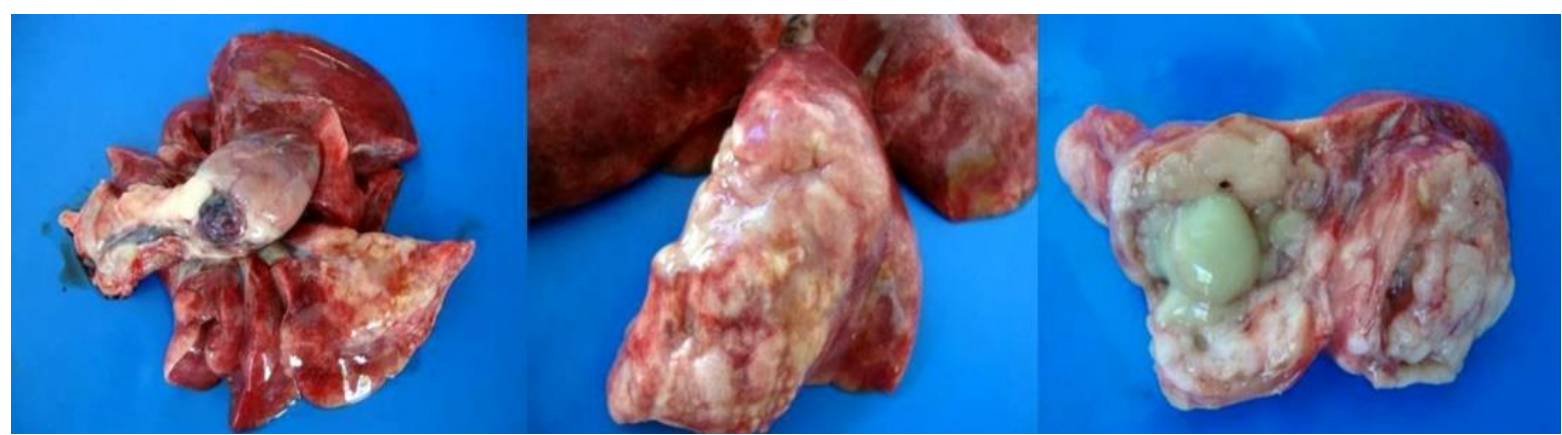

Figura 2. Felino, pulmão. Adenocarcinoma pulmonar. Lobo pulmonar caudal direito com massas multifocais a coalescentes, multilobados a cístico contendo muco.

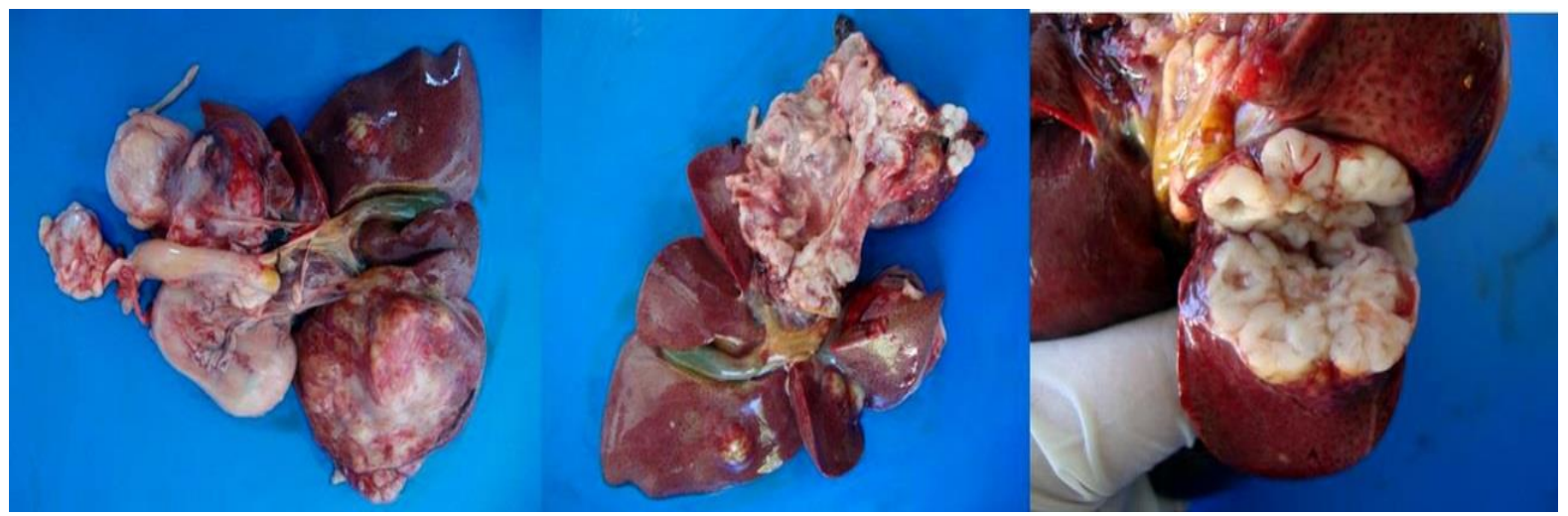

Figura 3. Felino, Fígado. Metástase de adenocarcinoma pulmonar em fígado. Nódulos e massa multifocal a coalescente em diferentes lobos, multilobados a cístico.

Os resultados citopatológicos das amostras pulmonares apresentavam acentuada população de células epiteliais cúbicas com moderada anisocariose, em arranjos trabeculares ou densos agregados celulares, com fundo contendo material sugestivo de muco (Figura 4). Já as amostras hepáticas apresentaram duas populações distintas, consistindo em hepatócitos isolados bem diferenciados e acentuada população de células epiteliais com morfologia semelhante à amostra pulmonar (Figura 5).

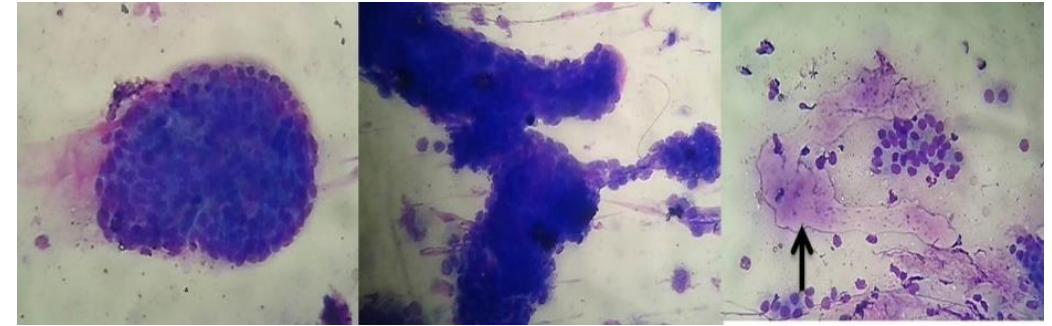

Figura 4. Felino, citologia de nódulo pulmonar com acentuada celularidade de células epiteliais neoplásicas arranjadas em trabéculas ou em densos agregados celulares com presença de muco ao fundo (seta) (Panótico, 40x).

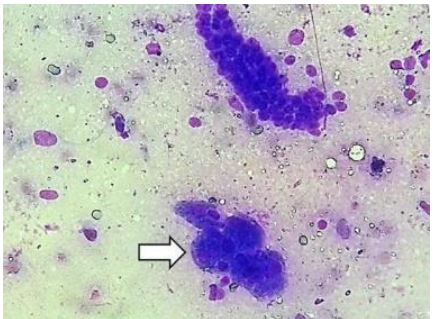

Figura 5. Felino, citologia de nódulo hepático, com acentuada celularidade de células epiteliais arranjadas em trabeculares e hepatócitos bem diferenciados isolados (seta branca). (Panótico, 40x).

O exame histopatológico do pulmão evidenciou acentuada proliferação de células neoplásicas de origem epitelial, com moderado pleomorfismo e anisocariose, com núcleo oval vacuolizado em arranjos sólidos, trabeculares, tubulares ou acinares separados por estroma fibrovascular (Figura 6). O fígado apresentava proliferação de células neoplásicas de origem epitelial com moderado pleomorfismo e anisocariose com núcleos ovais vacuolizados em arranjos tubulares e trabeculares se infiltrando no 
parênquima hepático normal (Figura 7). Não foram observadas alterações dignas de nota nos demais órgãos analisados.

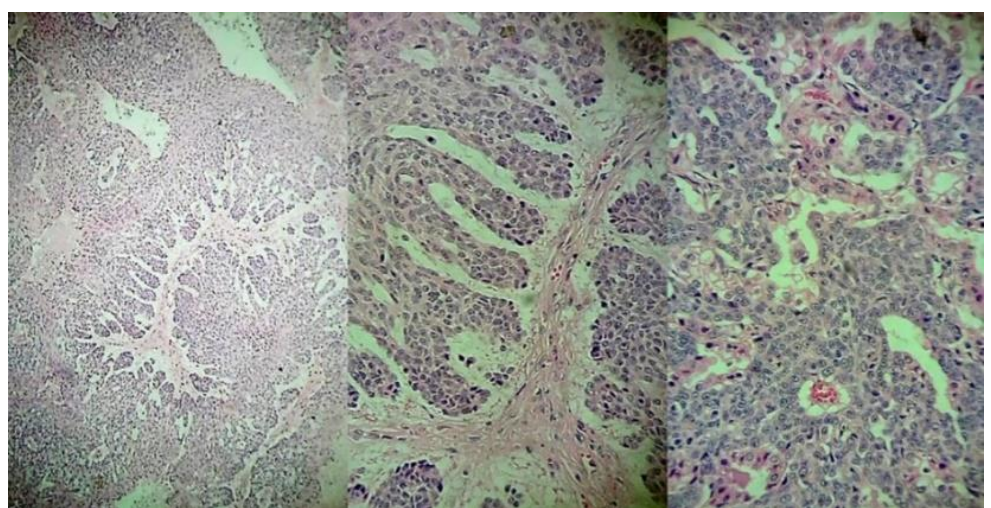

Figura 6. Felino, histologia de nódulo pulmonar com células neoplásicas em arranjos sólidos, trabeculares, tubulares ou acinares separados por estroma fibrovascular, apresentando moderado pleomorfismo (HE, 10x; 20x e 40x, respectivamente).

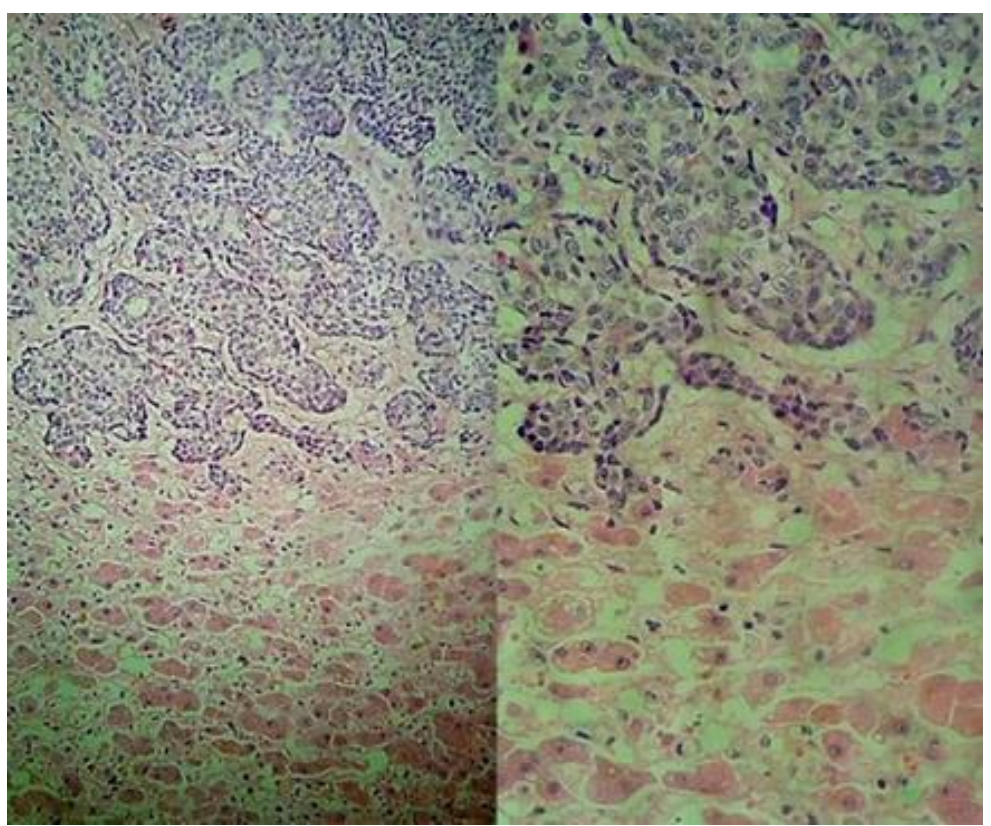

Figura 7. Felino, histologia de nódulo hepático com proliferação de células neoplásicas cúbicas com moderado pleomorfismo e anisocariose em arranjos trabeculares e acinares se infiltrando no parênquima hepático normal (HE, 20x, 40x, respectivamente).

Os resultados citopatológicos sugeriram o diagnóstico de carcinoma com aspectos morfocitológicos semelhantes em ambos os tecidos, sendo necessário a correlação clínica e radiológica para determinar a origem tumoral. Os achados citopatológicos foram compatíveis com o diagnóstico anatomopatológico de adenocarcinoma pulmonar misto, grau 2 (Wilson, 2016), com metástase hepática (HE, 20x, 40x, respectivamente).

\section{Discussão}

No presente relato, a idade e os sintomas clínicos de anorexia, perda progressiva, letargia e dispneia leve, associada a efusões pleurais e abdominais são condizentes com a literatura (Caswell \& Williams, 2016; Nunley et al., 2015).

Gatos domésticos apresentam uma incidência de neoplasia primária de pulmão superior às demais espécies, o que representa $0,75 \%$ de todas as condições patológicas em felinos idosos (Wilson, 2016) e afeta principalmente animais adultos com idade próxima aos 12 anos, sem predileção de sexo ou raça 
(Moulton et al., 1981). Em um estudo retrospectivo entre 1965 e 2008, 85,5\% dos tumores malignos de felinos acometiam o trato respiratório (Graf et al., 2016).

Hahn \& McEntee (1997) em seu estudo retrospectivo com 86 casos de tumores pulmonares primários em gatos, demonstram que sinais inespecíficos são os principais encontrados na clínica; enquanto que, sinais respiratórios, como dispneia, podem ocorrer em menos de 1/3 dos gatos acometidos, estando geralmente relacionadas a acometimentos pleurais ou crescimento tumoral.

A radiografia simples é essencial no diagnóstico e estadiamento clinico TNM (tumor, linfonodos e metástases) dos tumores pulmonares (Da Silva et al., 2012), sendo capaz de identificar e avaliar neoplasias, metástases e linfadenomegalia traqueobrônquica (Caprioli et al., 2018; Nunley et al., 2015).

Os achados radiográficos com massa única em lobo caudal associado à opacificação intersticial difusa (Figura 1; Tabela 1) são condizentes com os achados anatomopatológicos de efusão pleural e nódulos pulmonares coalescentes, acometendo $2 / 3$ do lobo caudal. Estes achados são corroborados pela a literatura que afirma que, a maioria dos carcinomas se apresentam como massas solitárias principalmente localizadas no lobo caudal, podendo estar associado à presença de efusão pleural e linfadenomegalia traqueobrônquica (Aarsvold et al., 2015; Romanucci et al., 2018). Comparado com achados radiográficos em humanos, a distribuição dos tumores felinos é menos consistente e apresenta pouca correlação histopatológica, sendo indicado a utilização de exames complementares como tomografia computadorizada, coleta de amostras citológicas ou lobectomia cirúrgica seguido de análise histopatológica (Aarsvold et al., 2015).

No presente caso, a análise anatomopatológica da neoplasia do pulmão e fígado apresentam aspectos e descrições semelhantes, sendo necessária a correlação clínica e radiológica para determinar a origem tumoral, confirmada pela análise histopatológica, que determinou o pulmão como foco neoplásico primário e o fígado como foco de metástase.

O diagnóstico de adenocarcinoma pulmonar grau 2, de caráter moderado, com comportamento invasivo, corrobora com a literatura que afirma que os tumores pulmonares em gatos são agressivos, com características invasivas ou expansivas (Lopez, 2013). O grau de diferenciação tumoral apresenta correlação significativa com a sobrevida do animal, como observado em estudo com 21 gatos tratados com ressecção cirúrgica, onde gatos com tumores moderadamente diferenciados apresentavam sobrevida de 698 dias, enquanto que tumores pouco diferenciados apresentavam sobrevida de 75 dias (Hahn \& McEntee, 1997).

Apesar da literatura afirmar que os principais sítios de metástase são intrapulmonares, diversos órgãos, como linfonodos regionais, pleura, mediastino, ossos, coração, músculos esqueléticos, pele, baço e outros órgãos abdominais podem ser afetados (Corgozinho et al., 2015; Jubb et al., 2007).

O animal do atual caso não apresentava alterações em membros inferiores para a suspeita de Síndrome Digital Pulmonar Felina, um padrão metastático atípico em falanges distais, encontrado em neoplasias malignas pulmonares, principalmente em casos de adenocarcinomas brônquicos e bronquialveolares (Thrift et al., 2017).

A histopatologia é o critério ouro para o diagnóstico definitivo, classificação tumoral e a identificação de seus diferentes fenótipos (Da Silva et al., 2012). Contudo, a despeito da citopatologia ser incapaz de analisar a arquitetura tecidual, impedindo a identificação dos diferentes fenótipos tumorais (Sabattini et al., 2017), é capaz de predizer o tipo tumoral e o seu comportamento biológico, através da transcrição dos padrões citológicos em padrões histológicos (Masserodotti, 2006).

Essa concordância dos padrões foi observada no presente caso, onde os achados citológicos são compatíveis com os achados histológicos. Assim, em ambas as técnicas as células apresentavam moderado grau de pleomorfismo; além de arranjos semelhantes, sendo na citopatologia organizadas em arranjos trabeculares ou densos agregados celulares; enquanto na histopatologia eram organizadas principalmente em arranjos trabeculares apoiados em estroma fibrovascular ou densos arranjos sólidos; além da presença de muco em ambas as técnicas.

A citopatologia é um método minimamente invasivo, seguro, pouco doloroso e com poucos riscos de complicação (Dolka et al., 2018). Podendo ser usado como método diagnostico rápido e barato em 
procedimentos cirúrgicos e necroscópicos quando o médico veterinário não tem acesso fácil a histopatologia por congelação ou laboratórios de patologia.

A citopatologia pode oferecer informações acuradas relevantes que podem guiar o exame postmortem (Walker \& Going, 1994). Assim, como pode guiar o cirurgião durante o ato cirúrgico, apresentando uma acurácia de 97,3\% na diferenciação de neoplasias benignas e malignas (Kolte \& Satarkar, 2010).

A punção aspirativa por agulha fina (PAAF) é uma técnica segura com alta sensibilidade diagnóstica para tumores primários de pulmão, apresentando maior precisão que a análise do escarro, lavado brônquio-alveolares e lavado traqueobrônquico (Bhatia et al., 2004; DeBerry et al., 2002). Um estudo comparativo entre o diagnostico citológico e histológico de neoplasias pulmonares pela PAAF em cães e gatos, a citologia demonstrou concordância dos diagnósticos em $82 \%$, com $100 \%$ de especificidade e $77 \%$ de sensibilidade (DeBerry et al., 2002).

A PAAF é indicada na presença de massas ou enfermidades difusas no parênquima pulmonar (Ferian et al., 2006; Pavel et al., 2016), podendo ser realizado pela coleta transbronquica por broncoscopia (Bhatia et al., 2004; DeBerry et al., 2002), guiada por tomografia computadorizada (Gupta et al., 2015) ou por ultrassom (DeBerry et al., 2002).

Além do mais, a PAAF de fígado é indicada em casos de neoplasias, com boa especificidade e valores preditivos positivos, com eficiente classificação para carcinoma hepatocelular (Bynum et al., 2015; Sharkey et al., 2014a; Sharkey et al., 2014b), podendo ser realizada em casos de animais que não são candidatos ao procedimento cirúrgico; sendo a coleta associada a exames de imagem, como ultrassonografia (Barbhuiya et al., 2014).

\section{Conclusão}

As comparações realizadas neste estudo sugerem que a combinação de diferentes métodos diagnósticos com o intuito de fornecer informações úteis ao clínico para a triagem e detecção precoce de neoplasias é capaz de gerar um diagnóstico preciso. Assim, o exame radiológico é essencial para o diagnóstico e estadiamento clínico de neoplasias pulmonares, ao passo que a histopatologia é o critério ouro para o diagnóstico definitivo e classificação tumoral. Contudo, a citopatologia é uma ferramenta rápida, pouco invasiva e segura para o diagnóstico preliminar e durante atos cirúrgicos e necroscópicos quando não se tem acessível histopatologia por congelação ou laboratórios de patologia.

\section{Referências}

Aarsvold, S., Reetz, J. A., Reichle, J. K., Reichle, J. K., Jones, I. D., Lamb, C. R., Evola, M. G., Keyerleber M. A. \& Macrolf, A. J. (2015). Computed tomographic findings in 57 cats with primary pulmonary neoplasia. Veterinary Radiology \& Ultrasound, 56, 272-277. DOI:10.1111/vru.12240

Aragão, J. (2013). - Guia Brasileiro de Boas Práticas em Eutanásia em Animais - Conceitos e Procedimentos Recomendados, 2013. Brasília, Brasil.

Barbhuiya, M., Bhunia, S., Kakkar, M., Shrivastava, B., Tiwari, P. K. \& Gupta, S. (2014). Fine needle aspiration cytology of lesions of liver and gallbladder: Ananalysis of 400 consecutive aspirations. Journal of Cytology, 31, 20-24. DOI:10.4103/0970-9371.130634

Bhatia, A., Singh, N. \& Arora, V. K. (2004). A Perspective on cytology of lung cancer. Indian J Chest Dis Allied Sci, 46, 81-83.

Burkhard, M. J. \& Millward, L. M. (2011). Respiratory Tract. In: Raskin, R.E., Meyer D.J. (ed.) Canine and Feline Cytology: a Color Atlas and Interpretation Guide, 2nd, Saunders. St. Louis, Missouri, USA.

Bynum, J. P., Duffield, A. \& Ali, S. Z. (2015). Impact of flow cytometry in liver cytopathology. Acta Cytologica, 59, 51-60. DOI: https://doi.org/10.1159/000370042

Caprioli, R. A., Argenta, F. F., Hammerschmitt, M. E., Pereira, P. R., Lorenzo, C., Pavarini, S. P., Driemeier, D. \& Sonne, L. (2018). Achados patológicos e imuno-histoquímicos de neoplasmas pulmonares primários em caninos na região metropolitana de Porto Alegre, Rio Grande do Sul. Pesquisa Veterinária Brasileira, 38, 1151-1158. DOI: https://doi.org/10.1590/1678-5150-pvb-5206. 
Caswell, J. L. \& Williams, K. J. (2016) Respiratory system. In: Jubb, Kennedy, \& Palmer's (ed) Pathology of Domestic Animals, $6^{\text {a }}$ ed, v.2, Elsevier, St. Louis, Missouri, USA.

Corgozinho, K. B., Belchior, C., Caloeiro, M. A. F., Cunha, S. C. S. \& Souza, H. J. M. (2015) Bronchogenic Adenocarcinoma with Unusual Metastasis in Cat. Acta Scientiae Veterinariae, 43 (Suppl1), 94.

Da Silva, E. O., Green, K. T., Wasques, D. G. \& Reis, A. C. F., Bracanense, A. P. F. R. L. (2012). Tumor primário pulmonar metastático em três cães. Semina Ciências Agrárias, 33, 3271-3278. DOI: https://doi.org/10.5433/1679-0359.2012v33supl2p3271

DeBerry, J. D., Norris, C. R., Samii, V. F., Riffey, S. M. \& Almy, F. S. (2002). Correlation between fine-needle aspiration cytopathology and histopathology of the lung in dogs and cats. Journal of the American Animal Hospital Association, 38, 327-336. DOI: https://doi.org/10.5326/0380327

Dolka, I., Czopowicz, M., Gruk-Jurka, A., Wojtkowska, A., Sapierzynski, R. \& Jurka, P. (2018). Diagnostic efficacy of smear cytology and Robinson's cytological grading of canine mammary tumors with respect to histopathology, cytomorphometry, metástases and overall survival. PLoS ONE. 13, e0191595. DOI:10.1371/journal.pone.0191595

Dungworth, D. L., Hauser, B., Hahn, F. F. Wilson, D. W., Taenichen, T. \& Harkema, J. R. Histological classification of tumors of the respiratory system of domestic animals (1999). Washington, DC:Armed Forces Institute of Pathology in cooperation with the American Registry of Pathology and the World Health Organization Collaborating Center for Worldwide Reference on Comparative Oncology, (2nd series) ed Armed Forces Institute of Pathology, Washington, DC.

Ferian, P. E., Silva, E. F., Guedes, R. C., Torres, R. C. S. \& Carneiro, R. A. (2006). Diagnóstico citológico de neoplasia pulmonar por meio de lavado broncoalveolar em uma cadela: relato de caso. Arquivo Brasileiro de Medicina Veterinária e Zootecnia, 58, 776-780. DOI: https://doi.org/10.1590/s0102-09352006000500012

Graf, R., Gruntzig, K., Boo, G. \& Hassig, M. (2016). Swiss feline cancer registry 1965-2008: the influence of sex, breed and age on tumour types and tumour locations. Journal of Comparative Pathology, 154, 195-210. DOI: 10.1016/j.jcpa.2016.01.008

Gupta, N., Sekar, A. \& Rajwanshi, A. (2015). Role of FNAC, fluid specimens, and cell blocks for cytological diagnosis of lung cancer in the present era. Journal of Cytology, 32, 217-222. DOI: 10.4103/0970-9371.171219

Hahn, K. A. \& Mcentee, M. F. (1997). Primary lung tumors in cats: 86 cases (1979-1994). Journal of the American Veterinary Medical Association, 211, 1257-1260.

Johnston, W. W.\& Bossen, E. H. (1981). The cytopathological diagnosis of lung câncer during the years 1970 to 1974 , with a comparison between cytopathology and histopathology in the typing of lung cancer. Acta Cytol., 25, 499-505.

Kolte, S. S. \& Satarkar, R. N. (2010). Role of scrape cytology in the intraoperative diagnosis of tumor. Journal of Cytology, 27 (3): 86-90. DOI: 10.4103/0970-9371.71871

Lopez A. (2013). Sistema Respiratório, Mediastino e Pleuras. In: McGavin, M. D. \& Zachary, J. F. (ed) Bases Patológicas em Veterinária, $5^{\text {a }}$ ed. Elsevier Mosby. St Louis, Missouri, USA

Magalhaes, A. M., Ramadinha, R. R., Barros, C. S. L. \& Peixoto, P. V. (2001). Estudo comparativo entre citopatologia e histopatologia no diagnóstico de neoplasias caninas. Pesquisa Veterinária Brasileira, 21, 23-32. DOI: https://doi.org/10.1590/S0100-736X2001000100006

Masserodotti, C. (2006). Architectural patterns in cytology: correlation with histology. Veterinary Clinical Pathology, 35, 388-396. DOI: 10.1111/j.1939-165x.2006.tb00154.x

Moulton, J. E., Von Tscharner, C. \& Schneider, R. (1981) Classification of lung carcinomas in the dog and cat. Veterinary Pathology, 18, 513-528. DOI: https://doi.org/10.1177/030098588101800409.

Nunley, J., Sutton, J., Culp, W., Wilson, D, Coleman, K., Demianiuk, R., Schechter, A., Moore, G., Donovan, T. \& Schwartz, P. (2015). Primary pulmonary neoplasia in cats: assessment of computed tomography findings and survival. Journal of Small Animal Practice, 56, 651-656. DOI: https://doi.org/10.1111/jsap.12401 
Pavel, G., Mălăncuş, R. \& Condrea, M. (2016). Fine needle aspiration cytology (FNAC) of neoplasms in dogs and cats, with emphasis on differential diagnosis - a retrospective study. Bulletin UASVM Veterinary Medicine, 73, 116 - 126. DOI: https://doi.org/10.15835/buasvmen-vm:11815

Romanucci, M., Massimini, M. Aste, G., Defourny, S. V. P., Crisis, P. E., Boari, A. \& Della Salda, L. (2018). Diffuse pulmonary adenocarcinoma with micropapillary growth pattern in a cat. Journal of Comparative Pathology, 160, 34-38. DOI: 10.1016/j.jcpa.2018.02.006

Sabattini, S., Renzi, A., Buracco, P., Defourny, S., Garnier-Moiroux, M., Capitani, O. \& Bettini, G. (2017). Comparative Assessment of the Accuracy of Cytological and Histologic Biopsies in the Diagnosis of Canine Bone Lesions. Journal of Veterinary Internal Medicine-31, 864-871. DOI: $10.1111 /$ jvim. 14696

Sharkey, L. C., Seelig, D. M. \& Overmann, J. (2014a). All lesions great and small, Part 1: Diagnostic Cytology in Veterinary Medicine. Diagnostic Cytopathology, 42, 535-543. DOI: https://doi.org/10.1002/dc.23097

Sharkey, L. C., Seelig, D. M. \& Overmann, J. (2014b). All lesions great and small, Part 2: Diagnostic Cytology in Veterinary Medicine. Diagnostic Cytopathology, 42, 544-552. https://doi.org/10.1002/dc.23090

Thrift, E., Greenwell, C., Turner, A-L., Harvey, A. M., Maher, D. \& Malik, R. (2017) Metastatic pulmonary carcinomas in cats ('feline lung-digit syndrome'): further variations on a theme. Journal of Feline Medicine and Surgery Open Reports, 3, 2055116917691069. DOI: $\underline{10.1177 / 2055116917691069}$

Travis W.D.; Brambilia E.; Noguchi M.; Nicholson A.G., Geisinger, K., Yatabe, Y., Ishikawa, Y., Wistuba, I., Flieder, D. B., Franklin, W., Gazdar, A., Hasleton, P. S., Henderson, D. W., Kerr, K. M., Petersen, I., Roggli, V., Thunnissen, E. \& Tsao, M. (2013). Diagnosis of lung cancer in small biopsies and cytology: implications of the 2011 International Association for the Study of Lung Cancer/American Thoracic Society/European Respiratory Society classification. Archives of Pathology \& Laboratory Medicine, 137, 668-684. DOI: 10.5858/arpa.2012-0263-RA

Van Der Linde-Sipman, J. S. \& Van Den Ingh, T. S. (2000) Primary and metastatic carcinomas in the digits of cats. Veterinary Quarterly, 22, 141-145. DOI: https://doi.org/10.1080/01652176.2000.9695043

Walker, E. \& Going, J. J. (1994). Cytopathology in the postmortem room. Journal of Clinical Pathology, 47 (8): 714-717. Doi: $10.1136 /$ jcp.47.8.714

Wilson, D.W. (2016). Tumors of the Respiratory Tract. In: Meuten D. J. (ed.) Tumors in domestic animals, 5a ed. Wiley-Blackwell, Haboken, NJ, USA.

Lopez A. (2013). Sistema Respiratório, Mediastino e Pleuras. In: McGavin, M. D. \& Zachary, J. F. (ed) Bases Patológicas em Veterinária, $5^{\text {a }}$ ed. Elsevier Mosby. St Louis, Missouri, USA

Histórico do artigo:

Recebido: 8 de setembro de 2020 Aprovado: 16 de outubro de 2020 . Disponível online: 11 de janeiro de 2021.
Licenciamento: Este artigo é publicado na modalidade Acesso Aberto sob a licença Creative Commons Atribuição 4.0 (CC-BY 4.0), a qual permite uso irrestrito, distribuição, reprodução em qualquer meio, desde que o autor e a fonte sejam devidamente creditados. 